\title{
EFFECTS OF THE SEASONAL CYCLE ON SUPERROTATION IN PLANETARY ATMOSPHERES
}

\author{
Jonathan L. Mitchell ${ }^{1,2,3}$, Geoffrey K. Vallis ${ }^{4}$, and Samuel F. Potter ${ }^{5}$ \\ ${ }^{1}$ Department of Earth \& Space Sciences, University of California Los Angeles, Los Angeles, CA 90095, USA \\ 2 Department of Atmospheric and Oceanic Sciences, University of California Los Angeles, Los Angeles, CA 90095, USA \\ ${ }^{3}$ School of Natural Sciences, Institute for Advanced Study, Princeton, NJ 08540, USA \\ ${ }^{4}$ College of Engineering, Mathematics and Physical Science, Exeter University, Exeter, UK \\ 5 Atmospheric and Oceanic Sciences Program, Princeton University, NJ 08544, USA \\ Received 2013 December 2; accepted 2014 March 26; published 2014 April 30
}

\begin{abstract}
The dynamics of dry atmospheric general circulation model simulations forced by seasonally varying Newtonian relaxation are explored over a wide range of two control parameters and are compared with the large-scale circulation of Earth, Mars, and Titan in their relevant parameter regimes. Of the parameters that govern the behavior of the system, the thermal Rossby number (Ro) has previously been found to be important in governing the spontaneous transition from an Earth-like climatology of winds to a superrotating one with prograde equatorial winds, in the absence of a seasonal cycle. This case is somewhat unrealistic as it applies only if the planet has zero obliquity or if surface thermal inertia is very large. While Venus has nearly vanishing obliquity, Earth, Mars, and Titan (Saturn) all have obliquities of $\sim 25^{\circ}$ and varying degrees of seasonality due to their differing thermal inertias and orbital periods. Motivated by this, we introduce a time-dependent Newtonian cooling to drive a seasonal cycle using idealized model forcing, and we define a second control parameter that mimics non-dimensional thermal inertia of planetary surfaces. We then perform and analyze simulations across the parameter range bracketed by Earth-like and Titan-like regimes, assess the impact on the spontaneous transition to superrotation, and compare Earth, Mars, and Titan to the model simulations in the relevant parameter regime. We find that a large seasonal cycle (small thermal inertia) prevents model atmospheres with large thermal Rossby numbers from developing superrotation by the influences of (1) cross-equatorial momentum advection by the Hadley circulation and (2) hemispherically asymmetric zonal-mean zonal winds that suppress instabilities leading to equatorial momentum convergence. We also demonstrate that baroclinic instabilities must be sufficiently weak to allow superrotation to develop. In the relevant parameter regimes, our seasonal model simulations compare favorably to large-scale, seasonal phenomena observed on Earth and Mars. In the Titan-like regime the seasonal cycle in our model acts to prevent superrotation from developing, and it is necessary to increase the value of a third parameter-the atmospheric Newtonian cooling time- to achieve a superrotating climatology.
\end{abstract}

Key word: planets and satellites: atmospheres

Online-only material: color figures

\section{INTRODUCTION}

In a classical prescription, all diabatic heat sources in a model atmosphere are reduced to Newtonian cooling, that is, to a linear relaxation of the potential temperature, $\Theta$, to a prescribed profile $\Theta_{e}$ :

$$
\frac{\partial \Theta}{\partial t}=-\frac{\Theta-\Theta_{e}}{\tau_{r}} .
$$

The forcing profile, $\Theta_{e}$, is a function of latitude and height but is normally assumed constant in time, with a single timescale, $\tau_{r}$ (also normally a function of space but not time), parameterizing the rate of heating/cooling. By implication, the climate system is assumed to have a substantial reservoir of heat capacity that averages over the seasonal cycle of insolation that results from Earth's finite obliquity. This is a decent, first-order assumption for Earth, where the oceans have considerable thermal inertia that moderates the seasonal cycle; however, it precludes the study of seasonal climate phenomena such as the monsoon, in which the inter-tropical convergence zone (ITCZ) is carried tens of degrees off the equator over continents. Furthermore, many planetary atmospheres have very strong seasonal cycles, and the steady forcing profile completely fails for Mars and Titan, whose solid surfaces with low thermal inertia produce strong seasonal cycles even with an Earth-like obliquity.

Idealized studies of Earth's general circulation have long employed Newtonian cooling to approximate steady forcing of the dynamics, and indeed Held \& Suarez (1994) introduced a now-standard benchmark calculation based on this type of model forcing. The simulated states of independently developed numerical atmospheric models (general circulation models, or GCMs) forced in this manner can be compared to demonstrate whether or not the models converge to the same answer. Plumb \& Hou (1992) and others have studied seasonal conditions under the assumption that the climate system responds to seasonal changes in a quasi-steady fashion, i.e., the applied forcing is hemispherically asymmetric but steady in time. However, an idealized GCM forced by Newtonian cooling with a generalized seasonal cycle that could apply both to Earth and other planets has thus far not be developed, and that is one of the goals of this paper.

Time-dependent models of the Earth and planetary atmospheres do of course exist. Thus, for example, and motivated by the dynamics of Earth's monsoons, Schneider \& Bordoni (2008) developed a time-dependent Newtonian cooling temperature profile, $\Theta_{e}$, that carries the ITCZ tens of degrees off the equator. However, their forcing scheme was not general enough 
to be applied to the other terrestrial planets. Also, numerical simulations of Mars, Titan, and other planets (e.g., Haberle et al. 1993; Wilson 1997; Forget et al. 1999; Mitchell et al. 2006; Mitchell 2012; Newman et al. 2011; Rannou et al. 2006; Tokano et al. 2001; Schneider et al. 2012; Lebonnois et al. 2012) have usually focused on direct integration of radiative transfer, convection, and other column processes forced by seasonally varying insolation.

In a companion paper (Mitchell \& Vallis 2010, hereafter MV10), a steady forcing scheme was used to study a wide range of planetary circulation regimes, roughly spanning the parameter space from Earth-like to Titan-like. We identified the thermal Rossby number, Ro, as an important control parameter on the resulting wind structure in steady state. Superrotating wind states, with the equatorial atmosphere spinning faster than the planetary surface, only develop for values of Ro larger than unity. While superrotation could in principle be generated and maintained by either vertical or horizontal Reynolds stresses, a key finding of MV10 is that horizontal stresses dominate.

This paper builds on the results described in MV10, and our particular aim is to explore the importance of a seasonal cycle in determining whether an atmosphere will spontaneously develop superrotation. While this approach is quite distinct from planetspecific modeling studies, whose primary aim is realism with respect to observations, we nevertheless critique our idealized model simulations in the relevant parameter regimes against large-scale, seasonal phenomena observed on Earth, Mars, and Titan. In the latter step, our intent is (1) to demonstrate how a simplified modeling study can lead to fundamental physical insight into mechanisms that are often obscured by model complexity, and (2) to demonstrate that the contrasting circulation regimes of Earth, Mars, and Titan can be understood in a unified context by filling in the gaps between them while varying a minimal set of model parameters. Read (2011) has also looked at planetary regimes, but without trying to reduce the model behavior to a minimal number of parameters.

The paper is organized in the following way. In Section 2, we introduce the parameter controlling the seasonal cycle and present the simulated temperature fields as a function of our control parameters. In Section 3, we further analyze the suite of simulations and characterize their climatological behavior. Earth, Mars, and Titan are put into the context of our nondimensional numbers in Section 5, and the simulations are critiqued against observed large-scale, seasonal phenomena on these bodies. We conclude in Section 6. A full description of the model is relegated to a series of appendices.

\section{IDEALIZED SEASONS WITH TIME-DEPENDENT NEWTONIAN COOLING}

To derive a physically motivated prescription of a timedependent $\Theta_{e}$, we begin by deriving the surface temperature of a slab surface in radiative equilibrium with a specified pattern of time-dependent insolation, neglecting the back-radiation from the atmosphere. As insolation warms different areas of the surface, we assume that convection communicates the low-level heat source through the troposphere so that $\Theta_{e}$ follows a specified lapse rate. A threshold is then applied, so that temperatures nowhere drop below a specified value, which both produces an isothermal stratosphere $(200 \mathrm{~K}$ as in Held \& Suarez 1994) and keeps winter pole temperatures from falling to unrealistic levels. As the next section shows, surface temperatures of the forcing profile, $\Theta_{e}$, derived in this manner exactly follow the specified insolation pattern if there is negligible heat capacity in the slab surface. If the surface heat capacity is non-negligible, on the other hand, the seasonal cycle of $\Theta_{e}$ has the physically realistic feature that temperature changes are both reduced in amplitude and offset in phase from the insolation.

\subsection{Formulation of the Horizontal Structure of the Time-dependent Forcing Profile}

We adopt a forcing profile of insolation that varies in latitude, $\varphi$, as

$$
S_{e}=\operatorname{Re} S_{o}\left[1+\frac{\Delta_{S}}{3}\left(1-3 \sin ^{2} \varphi+6 \sin \varphi e^{i \varphi_{1}(t)}\right)\right],
$$

where $S_{o}\left[1+\Delta_{S} / 3\right]$ is the annual- and global-mean insolation, $\Delta_{S}$ parameterizes the fractional meridional insolation gradient, and $\mathrm{Re}$ denotes the real part, which we take to be implicit in the formulae that follow. The time-dependent term, $6 \sin \varphi e^{i \varphi_{1}(t)}$, represents the latitude of maximum diurnally averaged insolation. We split Equation (2) into annual-mean (with overbars) and variable (with primes) components:

$$
\begin{gathered}
S_{e}=\bar{S}+S^{\prime} \\
\bar{S}=S_{o}\left[1+\frac{\Delta_{S}}{3}\left(1-3 \sin ^{2} \varphi\right)\right] \\
S^{\prime}=2 S_{o} \Delta_{S} \sin \varphi e^{i \varphi_{1}(t)} .
\end{gathered}
$$

We introduce the seasonal cycle by setting $\varphi_{1}=\omega t$, where $\omega=$ $2 \pi / P$ is the orbital frequency that drives the peak insolation from one pole to the other in a year. We neglect atmospheric back-radiation and require equilibrium between the annualmean insolation and surface infrared radiation, $\bar{S}=\sigma \bar{T}_{s}^{4}$, where the annual-mean surface temperature is $\bar{T}_{s}$. Assuming $\Delta_{S} \ll 1$,

$$
\bar{T}_{s} \simeq T_{o}\left[1+\frac{\Delta_{S}}{12}\left(1-3 \sin ^{2} \varphi\right)\right]
$$

where $T_{o}=\left(S_{o} / \sigma\right)^{1 / 4}$. The surface temperature has the desirable feature that annual-mean forcing profile temperatures are identical, regardless of the strength of the seasonal cycle (see Figure 2).

Next, we assume a surface with heat capacity per unit area, $C$, to be approximately in radiative equilibrium with the insolation and neglect the back-radiation from the atmosphere,

$$
C \frac{d T_{s}}{d t}=S-\sigma T_{s}^{4}
$$

Time-mean radiative fluxes must balance, $\bar{S}=\sigma \bar{T}_{s}^{4}$, and so linearizing about $\bar{T}_{s}$, we arrive at an equation for the timedependent surface temperature perturbation,

$$
\frac{d T_{s}^{\prime}}{d t}=\frac{S^{\prime}}{C}-\frac{T_{s}^{\prime}}{\tau_{f}}
$$

where we have introduced the thermal inertia of the surface as the timescale

$$
\tau_{f}=\frac{C}{4 \sigma \bar{T}^{3}} .
$$



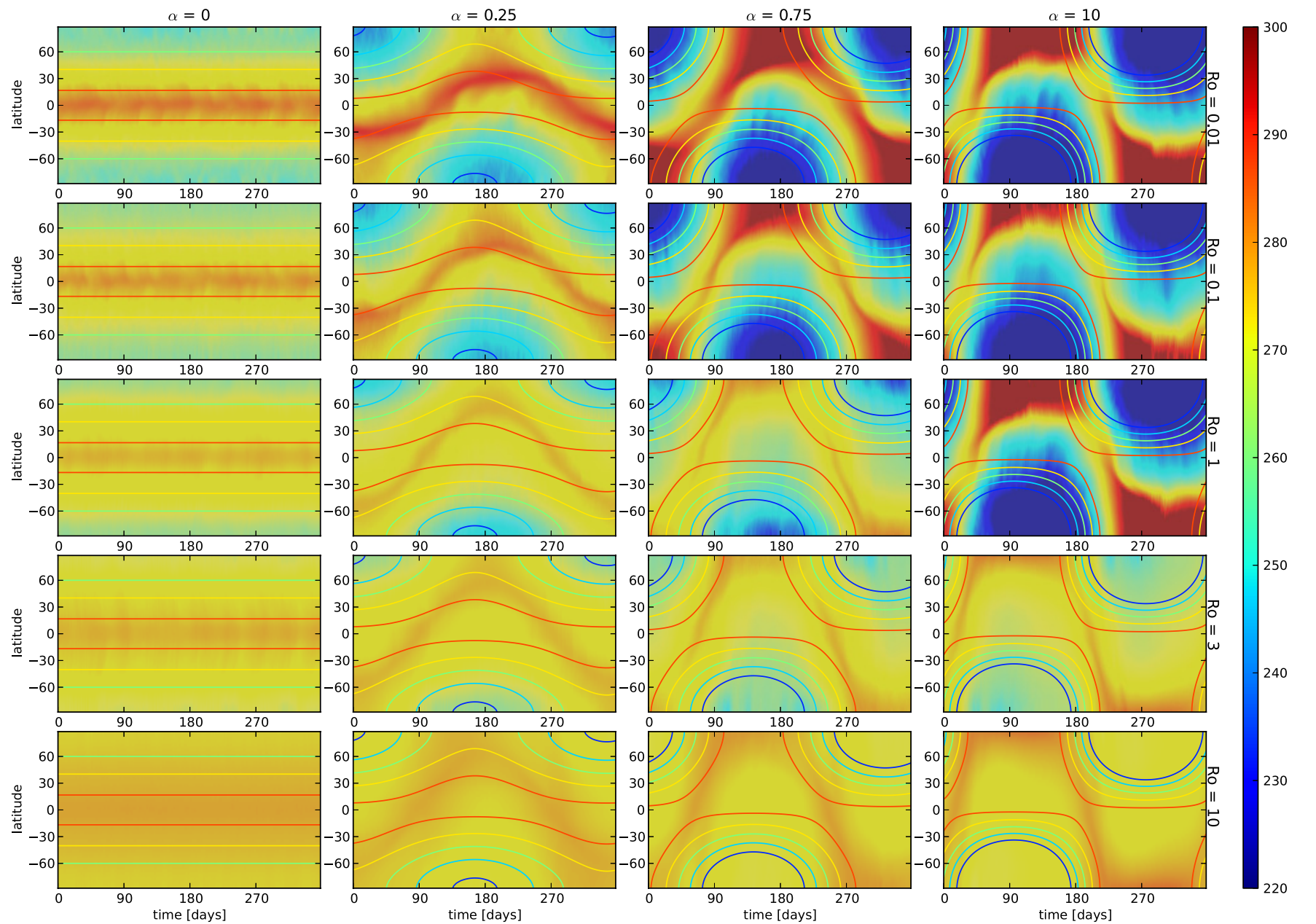

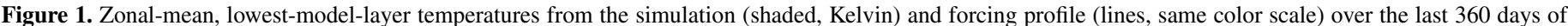

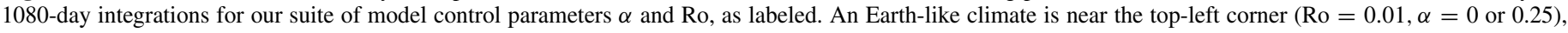

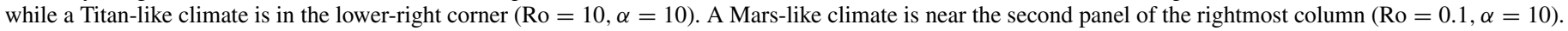

(A color version of this figure is available in the online journal.)

We non-dimensionalize with $t=t^{*} / \omega, S^{\prime}=S_{o} S^{\prime *}$, and $T_{s}^{\prime}=T_{o} T_{s}^{\prime * *}$; defining $\alpha=\left(\omega \tau_{f}\right)^{-1}$, the perturbation, nondimensional surface energy equation is (dropping the asterisks)

$$
\frac{d T_{s}^{\prime}}{d t}+\alpha T_{s}^{\prime}=\alpha \frac{\Delta_{s}}{2} \sin \varphi e^{i t},
$$

where we have made use of the identity $S_{o}=\sigma T_{o}^{4}$. Equation (10) has the solution

$$
T_{s}^{\prime}=\alpha \frac{\Delta_{S}}{2} \frac{\sin \varphi}{i+\alpha} e^{i t} .
$$

The time dependence of surface temperatures is controlled by a non-dimensional surface thermal inertia, $\alpha \equiv\left(\omega \tau_{f}\right)^{-1}$. In dimensional form, the solution is

$$
\begin{aligned}
T_{s}= & \max \left\{T^{*}, T_{o}\left[1+\frac{\Delta_{H}}{3}\left(1-3 \sin ^{2} \varphi\right)\right.\right. \\
& \left.\left.+2 \Delta_{H} \frac{\alpha \sin \varphi}{i+\alpha} e^{i \omega t}\right]\right\},
\end{aligned}
$$

where $\Delta_{H}=\Delta_{S} / 4$. As in Held \& Suarez (1994), we have applied a threshold, $T^{*}$, so that temperatures do not drop to unreasonable values in the winter hemisphere. There are two non-dimensional numbers from our derivation based on the surface energy equation: $\alpha$ and $\Delta_{H}$. Because Earth, Mars, and
Titan all have similar obliquities (relative to the ecliptic), we hold $\Delta_{H}$ fixed. Note that our derivation allows specification of surface thermal inertia through $\alpha$ in a manner that self-consistently introduces time-dependent temperature perturbations and their attendant phase shift relative to the seasons.

A range of scenarios is represented by the lines in Figure 1. If the thermal inertia timescale, $\tau_{f}$, is long compared to a year (e.g., $\alpha=0.25$ in the second column of Figure 1), the surface temperature approximately follows $T_{s}=T_{o}(1-$ $\left.\alpha 2 \Delta_{H} \sin \varphi e^{i(\omega t+\pi / 2)}\right)$. In this case, temperature perturbations lag the forcing by $90^{\circ}$, and their amplitude is reduced by a factor of $1 / \alpha$. In the opposite limit (e.g., $\alpha=10$ in the right column of Figure 1), $T_{s}=T_{o}\left(1+2 \Delta_{H} \sin \varphi e^{i \omega t}\right)$, and the forcing profile is in lockstep with the variation of the latitude of maximum insolation. Intermediate regimes have muted seasonal cycles and phase lags (e.g., $\alpha=0.75$ ).

\subsection{Specification of the Vertical Structure of the Forcing Profile}

Using the surface temperatures from Equation (12), we specify the stratification of the Newtonian cooling forcing profile by setting it to be a specified deviation from dry adiabatic (as in MV10), $\Delta \Gamma$, where

$$
\Delta \Gamma=\Gamma_{d}-\Gamma=\frac{g}{c_{p}}+\frac{d T}{d z},
$$



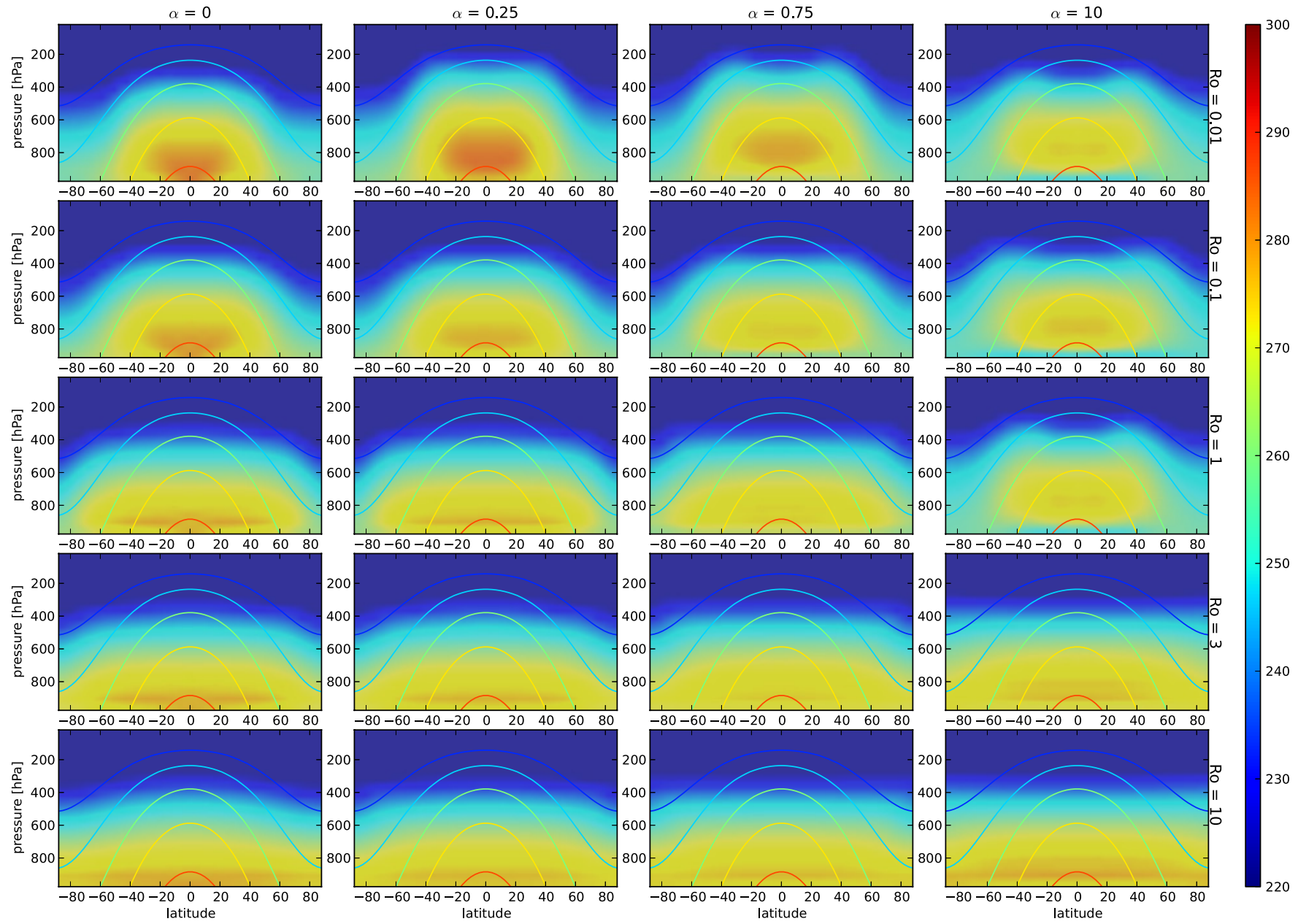

Figure 2. Zonal- and time-mean (days 721-1080) model temperatures (shaded, Kelvin) and forcing temperature profile (lines) arranged as in Figure 1.

(A color version of this figure is available in the online journal.)

where $\Gamma \equiv-d T / d z$ is the lapse rate and $\Gamma_{d} \equiv g / c_{p}$ is the dry adiabatic lapse rate, that is, the lapse rate that is convectively stable in a dry atmosphere. It is convenient to define the potential temperature (a measure of entropy)

$$
\theta=T\left(\frac{p_{o}}{p}\right)^{\kappa}
$$

and it is easily shown (e.g., Vallis 2006) that the dry adiabatic lapse rate corresponds to a profile with $\partial \theta / \partial z=0$. The radiative equilibrium profile is then specified to be

$$
T_{e}(t)=\max \left\{T^{*}, T_{s}(t)\left(\frac{p}{p_{o}}\right)^{\kappa\left(1-\Delta \Gamma / \Gamma_{d}\right)}\right\} .
$$

If the forcing temperature were to be dry adiabatic, we would set $\Delta \Gamma=0$, but the atmospheres of Earth, Mars, and Titan are a little more stable than that, so we choose $\Delta \Gamma / \Gamma_{d}=0.6$. Here the minimum temperature, $T^{*}$, serves the purpose of capping the troposphere with an isothermal stratosphere.

Tables 1 and 2 summarize the model forcing scheme and parameters for the numerical experiments in this study. Figure 1 displays the zonal-mean, lowest-model-layer temperature of the forcing profile (lines) and the model (shading; all on the same color scale to facilitate comparison) over the last 360 days of 1080-day integrations. The simulations are arranged from left to right by increasing seasonality, $\alpha$, and from top to bottom by increasing thermal Rossby number defined as in MV10, Ro =
$R \Delta_{H} /(2 \Omega a)^{2}$. Simulated latitudinal temperature gradients are largest at small Ro and large $\alpha$. Note, however, that although a large $\alpha$ imposes a strong latitudinal temperature gradient (lines), it does not guarantee large simulated temperatures gradients; a large Ro simulation (bottom row) always has weak temperature gradients. This is a result of an expansion of efficient lateral heat transport of the tropics that creates a global "weak temperature gradient" regime (Sobel \& Bretherton 2000). Figure 2 displays the time- and zonal-mean temperature of the forcing profile (lines) and the model simulations averaged over the last 360 days of 1080-day integrations (shading), arranged as in Figure 1. We note that the time- and zonal-mean forcing temperatures are identical for all cases (by construction). There is a clear progression toward a more barotropic state, with isotherms aligned horizontally, as Ro is increased (down columns). A stronger seasonal cycle ( $\alpha$ increasing from left to right) produces more complicated behavior in the annual mean. For instance, the Ro $=1$ (and perhaps the Ro $=0.1$ ) case appears to have enhanced latitudinal temperature gradients (more baroclinicity) for larger values of $\alpha$. This feature may be the result of enhanced winter-hemisphere temperature gradients, as is the case for Earth and Mars. The relevance of strong winter-hemisphere temperature gradients for Mars' seasonal cycle is discussed in Section 5.2.

Tables 3 and 4 display non-dimensional and dimensional parameters, respectively, for Earth and Titan. Roughly speaking, an Earth-like climate is near the top-left corner of Figures 1 
Table 1

Summary of Idealized Time-dependent Forcing

\begin{tabular}{c}
\hline \hline$\frac{\text { Equations for Idealized Momentum and Thermal Forcing }}{\frac{\partial(u, v)}{\partial t}=\cdots-r(\sigma)(u, v)} \quad \frac{\partial T}{\partial t}=\cdots-\tau_{r}(\varphi, \sigma)^{-1}\left(T-T_{e}(\varphi, \sigma)\right)$ \\
$T_{e}=\max \left\{T^{*}, T_{o}\left[1+\frac{\Delta_{H}}{3}\left(1-3 \sin ^{2} \varphi\right)+2 \Delta_{H} \frac{\alpha \sin \varphi}{i+\alpha} e^{i \omega t}\right] \sigma^{\kappa\left(1-\Delta \Gamma / \Gamma_{d}\right)}\right\}$ \\
$r=k_{f} \max \left(0, \frac{\sigma-\sigma_{b}}{1-\sigma_{b}}\right) \quad \tau_{r}^{-1}=k_{a}+\left(k_{s}+k_{a}\right) \max \left(0, \frac{\sigma-\sigma_{b}}{1-\sigma_{b}}\right)$ \\
$\sigma=p / p_{0}$
\end{tabular}

Constants

$k_{f}=1$ day $^{-1}$

$k_{a}=40$ days

$k_{s}=4$ days

$T_{0}=285 \mathrm{~K}$

$T_{o} \Delta_{H}=60 \mathrm{~K}$

$T^{*}=200 \mathrm{~K}$

$\kappa=2 / 7$

$\sigma_{b}=0.7$

$\Delta \Gamma / \Gamma_{d}=0.6$

$\Omega=7.3 \times 10^{-5} \mathrm{~s}^{-1}$

$p_{0}=1000 \mathrm{mb}$

$\omega=2 \pi / 360$ days $^{-1}$

Constants that recreate an Earth-like circulation: $(\operatorname{Ro}, \alpha) \sim(0.01,0)$

$a=6.4 \times 10^{6} \mathrm{~m} \quad \alpha=0$

Constants that recreate a Titan-like circulation: $(\operatorname{Ro}, \alpha) \sim(10,10)$

$a=2.8 \times 10^{5} \mathrm{~m} \quad \alpha=10 \quad k_{a}=4000$ days, $k_{s}=300$ days

$$
k_{f}=1 / 10 \text { days }^{-1}
$$

Table 2

Experiment Design, Keeping Earth-like Rotational $\left(\Omega=7.27 \times 10^{-5} \mathrm{~s}^{-1}\right)$ and Orbital Frequencies $\left(\omega=2 \times 10^{-7} \mathrm{~s}^{-1}\right)$ Fixed

\begin{tabular}{|c|c|c|c|}
\hline Ro & $\begin{array}{c}a \\
(\mathrm{~km})\end{array}$ & $\alpha$ & $\begin{array}{l}\tau_{f} \\
(\mathrm{~s})\end{array}$ \\
\hline 10 & 280 & 0 & $10^{20}$ \\
\hline 10 & 280 & 0.25 & $2 \times 10^{7}$ \\
\hline 10 & 280 & 0.75 & $6.7 \times 10^{6}$ \\
\hline 10 & 280 & 10 & $5 \times 10^{5}$ \\
\hline 1 & 800 & 0 & $10^{20}$ \\
\hline 1 & 800 & 0.25 & $2 \times 10^{7}$ \\
\hline 1 & 800 & 0.75 & $6.7 \times 10^{6}$ \\
\hline 1 & 800 & 10 & $5 \times 10^{5}$ \\
\hline 3 & 512 & 0 & $10^{20}$ \\
\hline 3 & 512 & 0.25 & $2 \times 10^{7}$ \\
\hline 3 & 512 & 0.75 & $6.7 \times 10^{6}$ \\
\hline 3 & 512 & 10 & $5 \times 10^{5}$ \\
\hline 0.1 & 3200 & 0 & $10^{20}$ \\
\hline 0.1 & 3200 & 0.25 & $2 \times 10^{7}$ \\
\hline 0.1 & 3200 & 0.75 & $6.7 \times 10^{6}$ \\
\hline 0.1 & 3200 & 10 & $5 \times 10^{5}$ \\
\hline 0.01 & 6400 & 0 & $10^{20}$ \\
\hline 0.01 & 6400 & 0.25 & $2 \times 10^{7}$ \\
\hline 0.01 & 6400 & 0.75 & $6.7 \times 10^{6}$ \\
\hline 0.01 & 6400 & 10 & $5 \times 10^{5}$ \\
\hline
\end{tabular}

Table 3

Estimated Non-dimensional Numbers for Earth and Titan

\begin{tabular}{lrc}
\hline \hline & \multicolumn{1}{c}{ Earth } & Titan \\
\hline Ro & $2 \times 10^{-2}$ & 10.5 \\
$E$ & $1.6 \times 10^{-1}$ & $2.2 \times 10^{-3}$ \\
$\left(\tau^{*}\right)^{-1}$ & $3 \times 10^{-2}$ & $5 \times 10^{-4}$ \\
$\alpha$ & $3.5 \times 10^{-2}$ & $>10$ \\
\hline
\end{tabular}

Table 4

Dimensional Parameters for Dynamical Regimes Similar to Earth and Titan

\begin{tabular}{lcc}
\hline \hline & Earth-like & Titan-like \\
\hline$\Omega\left(\mathrm{s}^{-1}\right)$ & $7.3 \times 10^{-5}$ & $4.5 \times 10^{-6}$ \\
$\omega\left(\mathrm{s}^{-1}\right)$ & $2 \times 10^{-7}$ & $6.7 \times 10^{-9}$ \\
$a(\mathrm{~km})$ & 6371 & 2575 \\
$\tau_{o}(\mathrm{~s})$ & $1.4 \times 10^{7}$ & $2.2 \times 10^{8}$ \\
$\tau_{f}(\mathrm{~s})$ & $2.9 \times 10^{4}$ & $8.64 \times 10^{5}$ \\
$r\left(\mathrm{~s}^{-1}\right)$ & $3.2 \times 10^{-7}$ & $2 \times 10^{-8}$ \\
$T_{o}(\mathrm{~K})$ & 285 & 94 \\
$T_{o} \Delta_{H}(\mathrm{~K})$ & 60 & 20 \\
\hline
\end{tabular}

and $2(\operatorname{Ro}=0.01, \alpha=0$ or 0.25$)$, while a Titan-like climate is in the lower-right corner ( $\operatorname{Ro}=10, \alpha=10)$. A Marslike climate is near the second panel of the rightmost column ( Ro $=0.1, \alpha=10)$. These particular cases are revisited in Section 5 .

\section{MEAN STATES AND PARAMETER REGIMES OF SIMULATIONS}

Figure 3 shows the zonal-mean zonal winds averaged over the last 360 days, arranged by parameter values as in Figure 1. Several trends are worth noting. First, wind speeds decrease with increasing $\alpha$ in nearly all cases (the one exception being at Ro $=0.01, \alpha=0$ ). This is to be expected as a strong, cross-equatorial flow at solstices causes westward acceleration at the equator. Superrotation develops as Ro increases, with the transition at Ro $=1$ identified in MV10. Figure 4 summarizes these results in a regime diagram of the vertical-mean equatorial wind. By focusing on the vertical mean equatorial wind, we isolate superrotation that develops as the result of horizontal momentum fluxes, not vertical ones (as, for instance, in the quasi-biennial oscillation, QBO). Somewhat surprisingly, a large region of the parameter space superrotates, although only a small fraction at low $\alpha$ does so strongly. The right panel shows a zoom of this region, which makes clearer the trend toward stronger superrotation for larger Ro.

Superrotation is not robustly established for large $\alpha$, apparently for two reasons: strong seasonality (1) reduces the average angular momentum of the atmosphere at all latitudes including the jets and (2) prevents eddy momentum flux convergence from accelerating equatorial winds. We discuss these two effects in detail below.

Under the influence of a strong seasonal cycle, the ITCZ experiences large excursions away from the equator. The Hadley cell is responsible for supplying the surface angular momentum that is transferred to the atmosphere in the frictional boundary layer into the frictionless free troposphere. Under steady forcing, the ITCZ stays fixed on the equator, where surface angular momentum is maximal. In seasonal cases, the ITCZ spends much of the time off the equator, where the surface angular momentum is lower than at the equator. As a result, the average angular momentum imparted to the free troposphere is smaller in a seasonal case than in a steady case (for discussion of this effect on Titan, see Mitchell 2008; Mitchell et al. 2009). Figure 5 displays the vertical-mean equatorial wind speed, $U_{\text {eq }}$ (top), and lowest-model-layer pressure velocity (bottom) over the last 360 days of the Ro $=10$ cases. Any positive value of $U_{\text {eq }}$ in the top row indicates superrotation. Warm colors in the lower row indicate the presence of upwelling by the ITCZ, and cold colors indicate downwelling. As $\alpha$ increases (to the right), the average value of $U_{\text {eq }}$ decreases and shows more seasonal variation. 

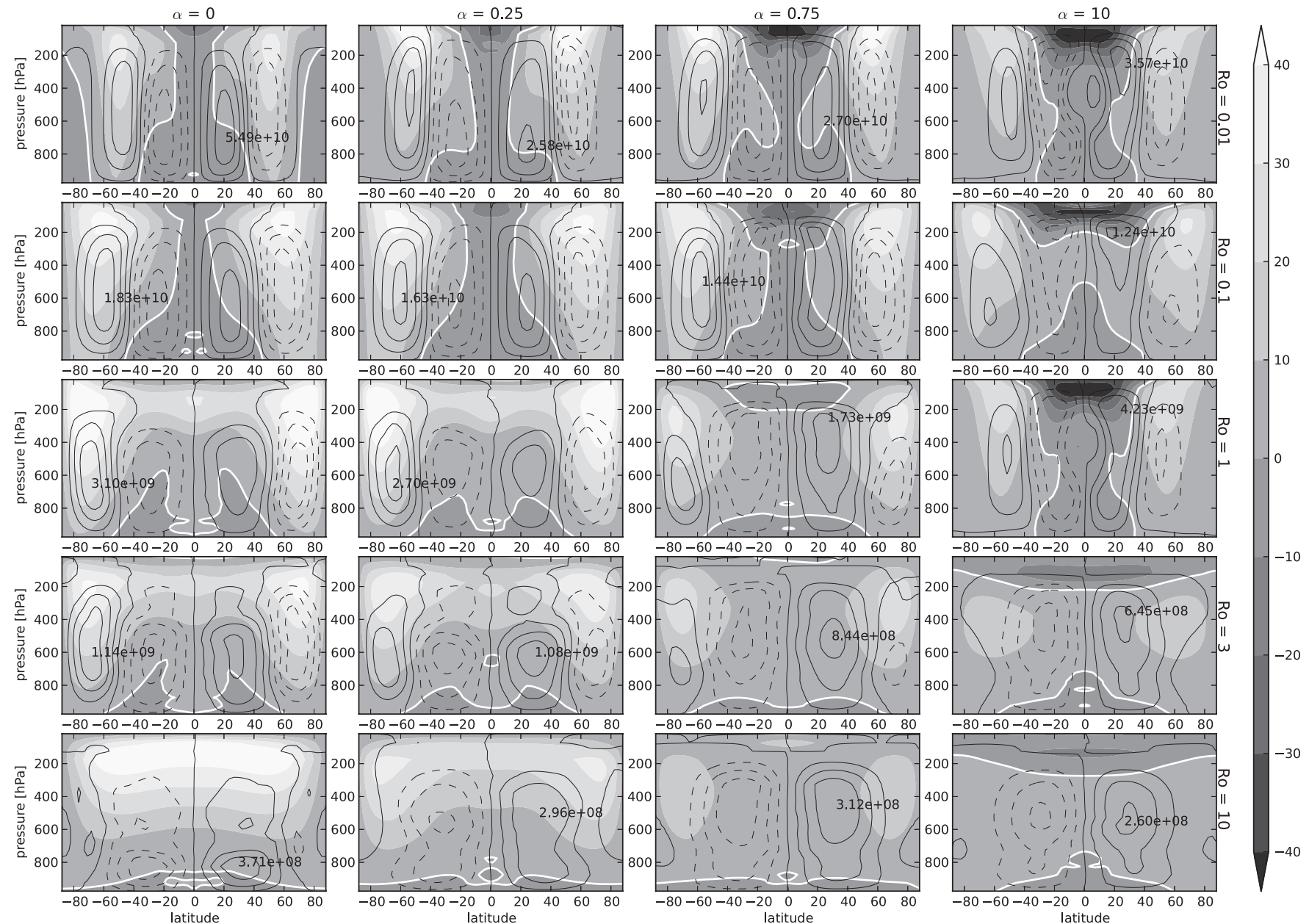

Figure 3. Zonal- and time-mean (days 721-1080) zonal winds (gray scale in $\mathrm{m} \mathrm{s}^{-1}$; zero-wind line marked with bold white) and overturning stream function (lines with maximum labeled in $\mathrm{kg} \mathrm{s}^{-1}$ arranged as in Figure 1).
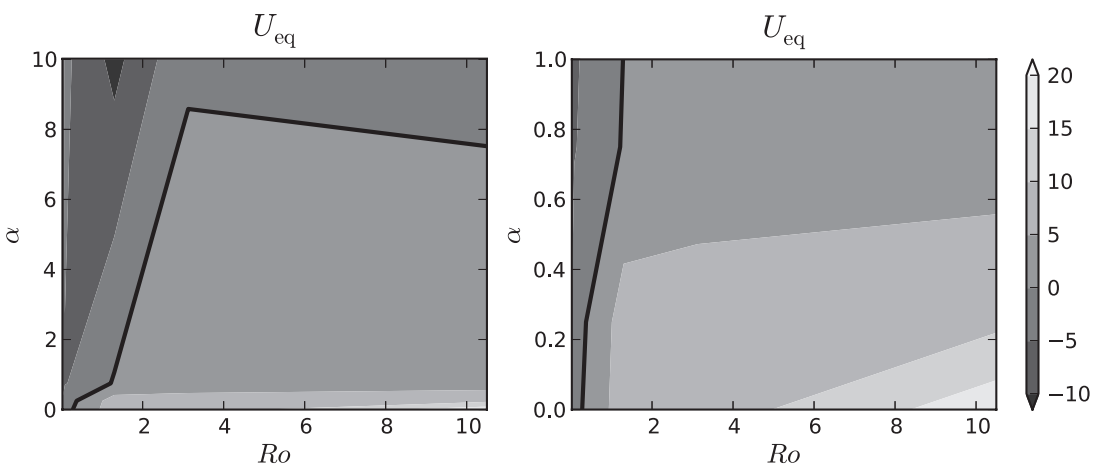

Figure 4. Vertical-, zonal-, and time-mean (days 721-1080) equatorial zonal winds, $U_{\text {eq }}$, arranged as in Figure 1 . The bold black line marks $U_{\text {eq }}=0$, the solid-body value. (The right panel is a zoom-in of the left panel along the $y$-axis.)

To understand the decreasing magnitude of $U_{\text {eq }}$, we appeal to the connection between the surface and atmosphere through friction. The surface, being a solid-body rotator, has a maximum value of angular momentum at the equator. Since the Hadley cell is responsible for communicating surface friction out of the boundary layer into the nearly frictionless free troposphere, the latitude of Hadley cell upwelling (ITCZ) determines the surface value of angular momentum that is imparted to the atmosphere. As the Hadley circulation diverges from this latitude at upper levels, air parcels conserve their angular momentum so that upper-level equatorward flow results in a deceleration of winds and poleward flow an acceleration of winds. Thus, a reduction in the average value of $U_{\text {eq }}$ is consistent with the transport of low angular momentum air into the free troposphere by the seasonally migrating ITCZ (although see Figure 12 for the influence of the strength of Hadley cell overturning).

The seasonal cycle in $U_{\text {eq }}$ itself may indicate seasonal variation in the eddy activity responsible for equatorial acceleration. To explore this further, Figure 6 shows the acceleration of the zonal-mean momentum by eddy flux convergence (a measure of the acceleration of the zonal-mean flow by horizontal Reynolds stresses), $\mathcal{S}=-\left(a \cos ^{2} \phi\right)^{-1} \partial_{\phi}\left(\overline{u^{\prime} v^{\prime}} \cos ^{2} \phi\right)$ (top), in the last 360 days of the seasonal Ro $=10$ cases; the zonalmean zonal wind, $\bar{u}$ (bottom), at the $400 \mathrm{hPa}$ level is shown for 

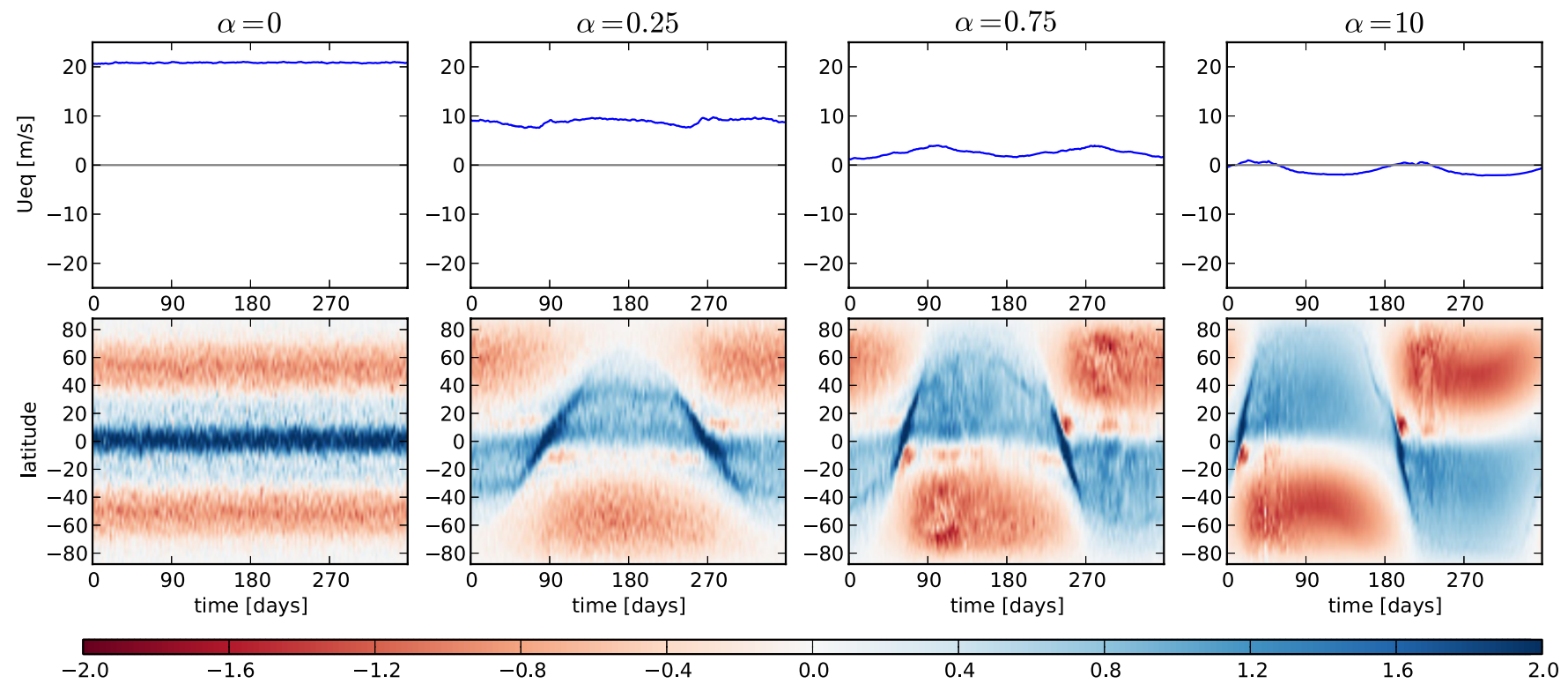

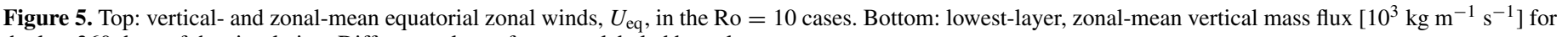
the last 360 days of the simulation. Different values of $\alpha$ are as labeled by column.

(A color version of this figure is available in the online journal.)
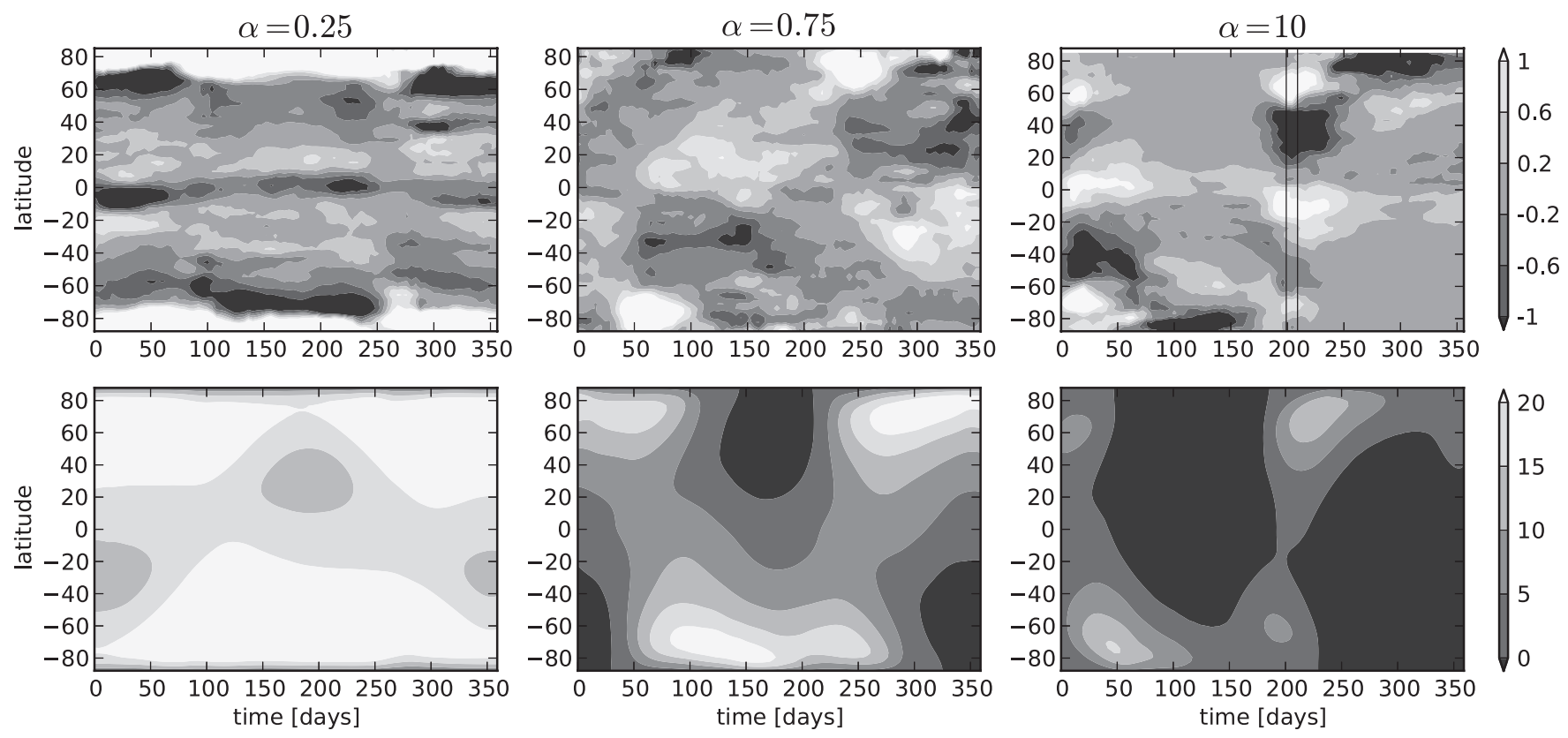

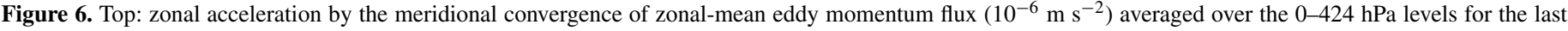

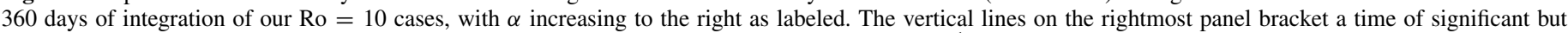

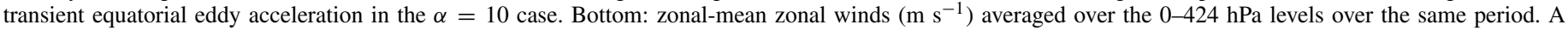
Gaussian smoothing kernel of six day half-width was applied to the time domain for all fields.

comparison. Henceforth, overbars indicate zonal means and primes departures from the zonal mean. In the presence of a seasonal cycle, this form of decomposition allows the possibility of time dependence/transience in both the eddy and mean components of the zonal momentum flux. As $\alpha$ is increased (to the right in Figure 6), a strong seasonal cycle becomes apparent in both $\bar{u}$ and $\mathcal{S}$, and the pattern and strength of $\mathcal{S}$ strongly correlate in time with $\bar{u}$. In fact, equatorial acceleration in strongly seasonal runs $(\alpha=10)$ is a unique feature of hemispherically symmetric conditions, when the zonal-mean zonal winds are nearly symmetric about the equator.

The transiency of equatorial eddy stresses during equinoxes motivates a closer inspection of these times. Figure 7 displays the eddy momentum flux convergence at $424 \mathrm{hPa}$ during days 200-210 (marked as vertical lines in the upper-right panel of Figure 6) as a function of latitude and phase speed of the zonally asymmetric modes in the Ro $=10, \alpha=10$ case. The zonal-mean zonal winds at this level are overplotted. This type of diagram allows one to inspect for critical lines, where the phase speed of waves giving rise to the eddy stresses is equal to the zonal-mean zonal wind. Quasi-linear theory suggests that the presence of critical lines causes waves to break and deposit their pseudomomentum into the mean flow (see MV10 for a full description). If the modes responsible for the eddy stresses were pure Rossby waves sourced at mid- or high latitudes, they could not have propagated much past the critical 


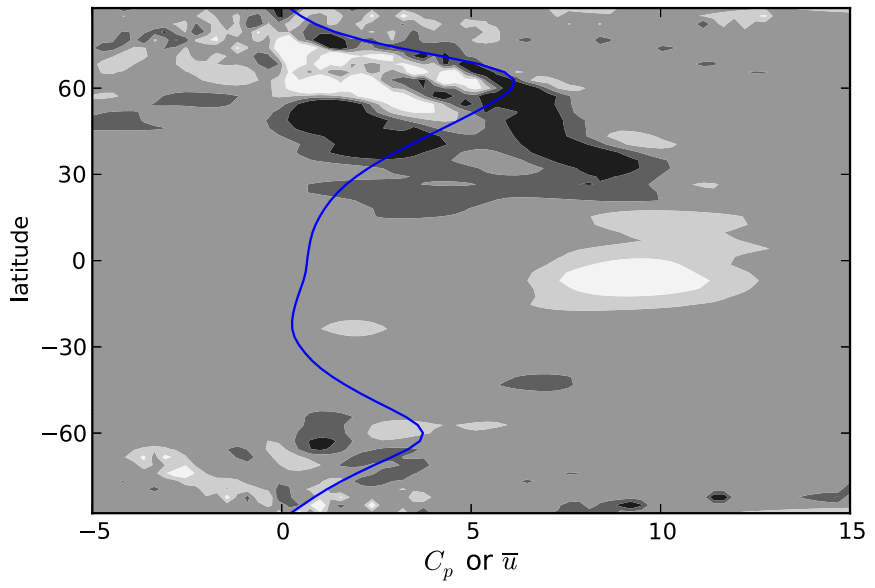

Figure 7. Convergence of the zonal-wavenumber-1 eddy momentum flux cospectrum as a function of latitude and phase speed averaged between 0 and $424 \mathrm{hPa}$ for the Ro $=10, \alpha=10$ case (acceleration in white) and zonalmean zonal winds (blue line) during days 200-210 (the 10-day span marked in Figure 6). The cospectrum is calculated by fast Fourier transform of the zonal and meridional winds in the 10 day interval centered on day 204 and at the $424 \mathrm{hPa}$ level, after Randel \& Held (1991).

(A color version of this figure is available in the online journal.)

lines at $\sim \pm 40^{\circ}$ latitudes. Instead, as in MV10, a coherent, global mode that spans both sides of the critical lines is present that decelerates mid- and high-latitude winds and accelerates equatorial winds, albeit with less global coherence than the mode in MV10. Figure 8 displays the $424 \mathrm{hPa}$ geopotential height ${ }^{6}$ and winds at day 205 filtered to retain only zonal-wavenumber 1 features, i.e., fluctuations that have one wavelength at each latitude circle. Interacting modes are clearly identifiable, a highlatitude Rossby-like wave and an equatorial Kelvin-like wave. The global mode has the same general appearance as the one identified in MV10 as being responsible for the acceleration of equatorial winds during spinup of the Ro $=10$ case (without seasons), except that it now has some hemispheric asymmetry. A significant implication is that equatorial superrotation may require a persistent zonal-mean state that is nearly symmetric about the equator.

Taken together, these results indicate an important role for the seasonal cycle in the development and maintenance of superrotation by (1) diluting the angular momentum supply to the free troposphere and (2) producing only transient phases at equinox with zonal-mean winds that allow the global mode in Figure 8 to accelerate equatorial winds.

\section{THE ROLE OF BAROCLINIC INSTABILITY}

Baroclinic instability is well known as the source of Earth's mid-latitude weather systems, and it arises in rotating systems in which there is a horizontal temperature gradient and a fairly mature theory exists for it (e.g., Pierrehumbert \& Swanson 1995; Vallis 2006). The instability converts potential energy into kinetic energy, and in so doing it generates Rossby waves that may propagate away from the disturbance. Wave mean-flow theory then describes how these Rossby waves may influence the zonal wind structure. In a quasi-linear perspective, Rossby waves generated in the mid-latitude baroclinic zone propagate equatorward and break in the subtropical surf zone, where

\footnotetext{
6 Geopotential height is the height in $m$ of a pressure surface above sea level calculated assuming a constant gravitational acceleration, $g$, and is proportional to an ln-pressure weighted, vertical integral of the temperature.
}

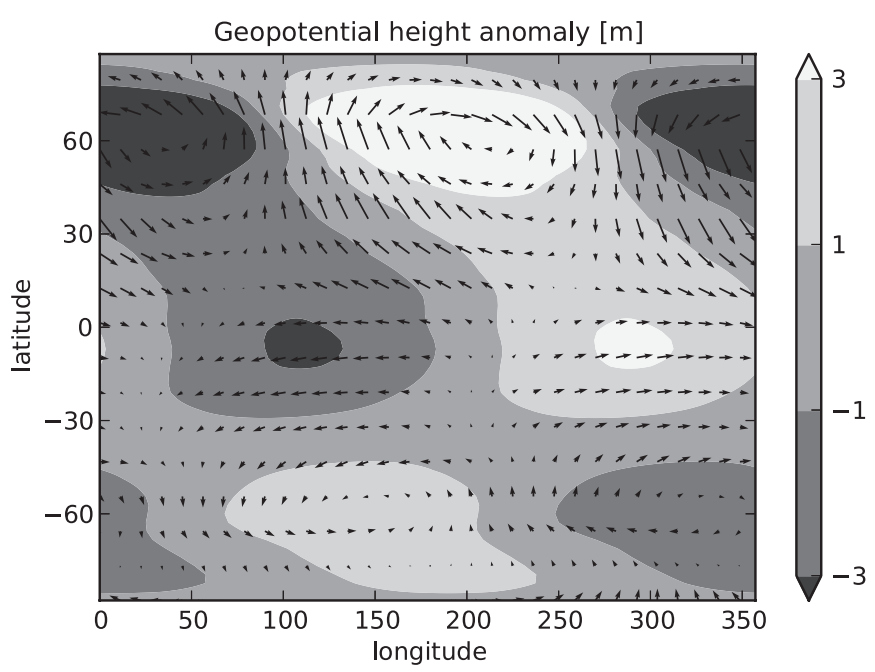

Figure 8. $424 \mathrm{hPa}$, zonal-wavenumber-1 geopotential height anomaly at day 205 of the $\mathrm{Ro}=10, \alpha=10$ case.

meridional shear creates a critical layer that prevents further wave propagation into the tropics (Randel \& Held 1991). The wave propagation and breaking effectively "suck" momentum from this subtropical region and deposit it in the mid-latitude jet. Because these waves provide a negative torque to the zonalmean zonal winds in the subtropics, their presence may prevent the development of equatorial superrotation (even if other instabilities are present that accelerate equatorial winds; Held 2000). In a superrotating state, however, the subtropical critical layer lifts and the tropics become "transparent" to propagating Rossby waves (see Figure 8 in MV10). If a mechanism existed to accelerate equatorial winds, even Earth's atmosphere might achieve a superrotating steady state, a concept that is supported by some idealized simulations (Suarez \& Duffy 1992; Saravanan 1993). Rossby waves generated by baroclinic instability in mid-latitudes otherwise inhibit the development of superrotation through their negative torque applied to the zonal-mean flow in the subtropical surf zone. We might, therefore, expect superrotation to be anti-correlated with baroclinicity.

Baroclinic supercriticality, $S_{C}=L_{\beta} / L_{R}$, is a measure of the nonlinearity of the inverse turbulent energy cascade from the Rossby deformation radius, $L_{R}$, to the Rhines scale, $L_{\beta}$, and it serves as a scalar measure of the degree of baroclinicity in our simulations. Figure 9 shows baroclinic supercriticality (SC) plotted against superrotation index SI, where

$$
\operatorname{SI}(t)=\frac{\int_{-\pi}^{\pi}\left([\bar{u}] \cos \varphi+\cos ^{2} \varphi\right) \cos \varphi d \varphi}{\int_{-\pi}^{\pi} \cos ^{3} \varphi d \varphi},
$$

for our suite of simulations. A clear anti-correlation is present, i.e., increasing degree of superrotation with decreasing baroclinic SC. Strong superrotation with SI $>1.2$ only exists in the subcritical regime $(\mathrm{SC}<1)$. Closer inspection of the nonseasonal cases reveals a monotonic trend toward increasing SI for increasing Ro. However, there is a non-monotonic trend in baroclinic SC with Ro; peak SC occurs at Ro $\sim 1$ and decreases to both larger and smaller values of Ro.

Therefore, in addition to a weak seasonal cycle, weak baroclinicity is a necessary but insufficient condition for the development of spontaneous superrotation. A weak Hadley cell also appears to be a necessary but insufficient condition (dias Pinto 


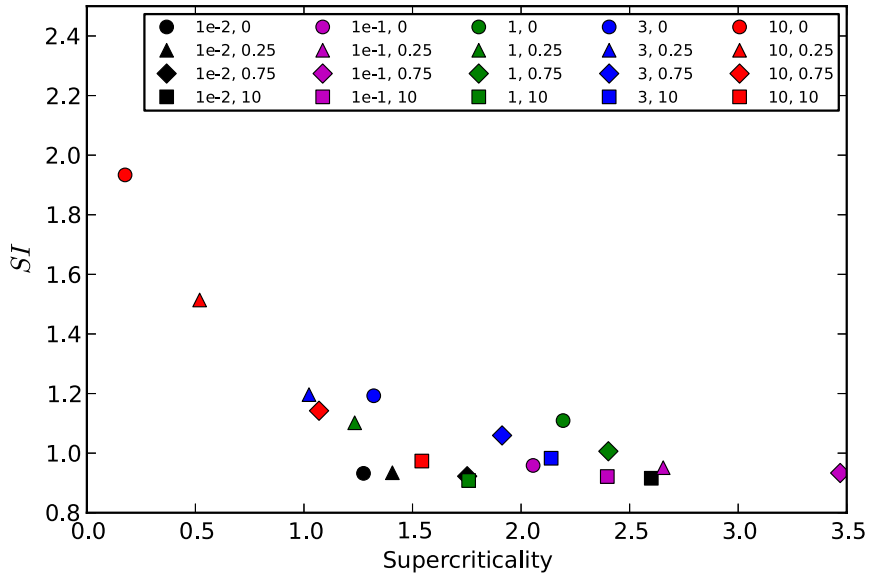

Figure 9. Superrotation index vs. the diagnosed baroclinic supercriticality (after Schneider 2004) averaged over the last 360 days of our simulations. Commaseparated values in the legend indicate the value of Ro and $\alpha$, respectively.

(A color version of this figure is available in the online journal.)

\& Mitchell 2014), but these anti-correlations do not illuminate what is responsible for equatorial acceleration. Simulations at intermediate Ro indicate a role for Kelvin-like waves in accelerating equatorial zonal winds, which brings into question the prevailing idea that barotropic instability is responsible for the generation of superrotation (Potter et al. 2013). What is obviously lacking is a description of the wave instability responsible for acceleration of equatorial zonal winds, and this is left to future work (P. Wang \& J. L. Mitchell 2014, in preparation).

\section{RELEVANCE TO SOLAR SYSTEM BODIES}

We now discuss the realism of our idealized calculations and how, in the appropriate parameter regimes, they relate, or fail to relate, to the solar system bodies of Earth, Mars, and Titan.

\subsection{Earth}

The summer monsoon is an important signal of Earth's seasonal cycle in the tropics, and it is interesting to see to what extent the model, a dry one, can reproduce aspects of it. As shown in Figure 10(b), the lowest-model-layer temperature forcing (colored lines) of our Ro $=0.01, \alpha=0.25$ case is similar to the seasonal cycle in (Schneider \& Bordoni 2008). Figure 10(a) displays the lowest-layer, zonal-mean vertical mass flux, and panel (b) shows the lowest-layer, zonal mean temperatures over the last 360 days of this simulation. The ITCZ migrates $20^{\circ}-30^{\circ}$ off the equator into the summer hemisphere; however, it does so in a somewhat smoother (in time) fashion than found in the abrupt, eddy-mediated monsoon transition seen in the Asian monsoon sector (Bordoni \& Schneider 2008) and in other idealized, dry simulations (Schneider \& Bordoni 2008). Inspection of the zonal-mean meridional stream function (black lines) and zonal-mean atmospheric angular momentum (gray lines) in Figure 10 during the summer monsoon reveals that the winter Hadley cell is far from angular momentum conserving (black and gray lines would otherwise be parallel). In reanalysis, the monsoonal circulation has its maximum value in the summer hemisphere and is close to angular momentum conserving (Bordoni \& Schneider 2008). This shortcoming of (a)

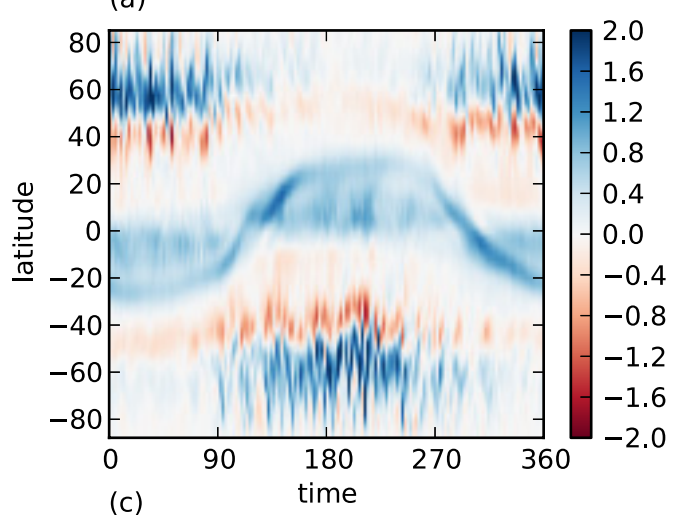

(b)

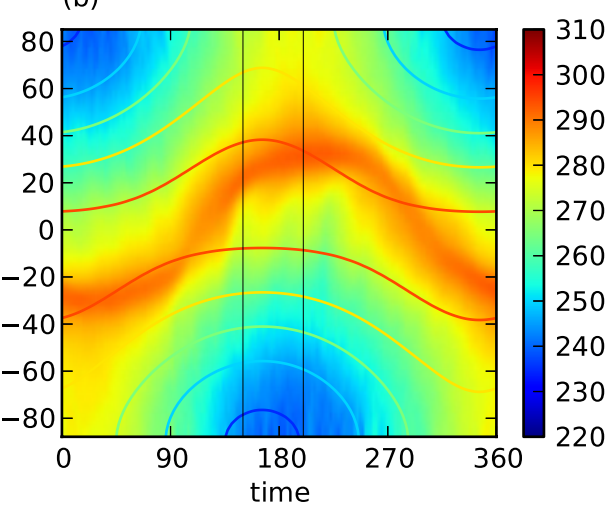

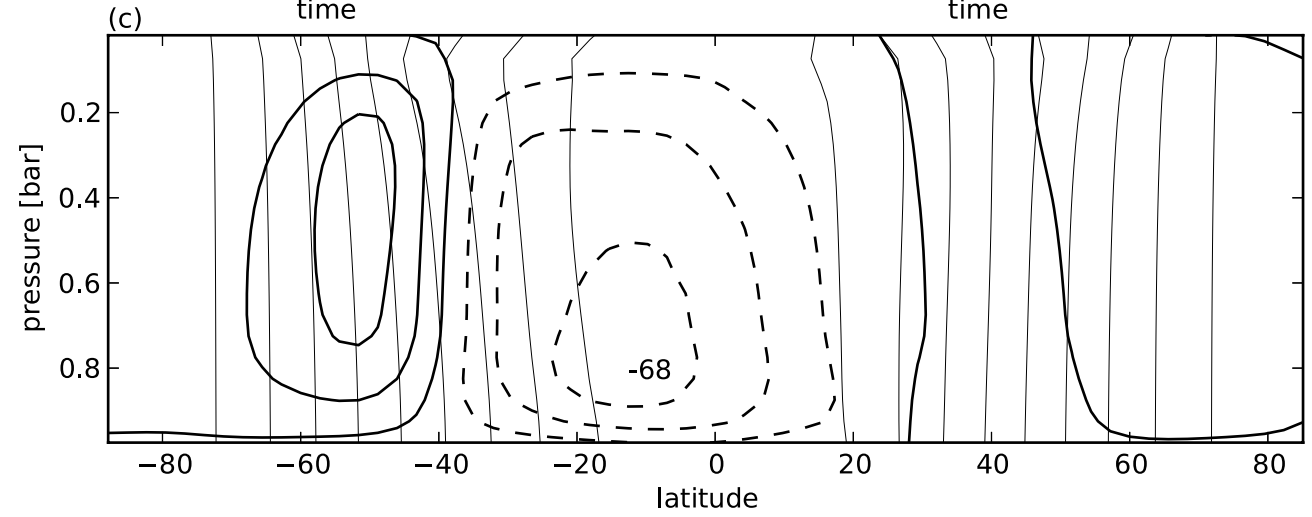

Figure 10. Earth-like Ro $=0.01, \alpha=0.25$ case. (a) Lowest-layer, zonal-mean vertical mass flux $\left[10^{4} \mathrm{~kg} \mathrm{~m}^{-1} \mathrm{~s}^{-1}\right]$ for the last 360 days of the simulation. (b) As in (a) for the zonal-mean, lowest-layer temperature. (c) Mean meridional stream function (black lines, $20 \times 10^{9} \mathrm{~kg} \mathrm{~s}^{-1}$ contour interval; minimum value labeled in $10^{9} \mathrm{~kg} \mathrm{~s}^{-1}$ ) and atmospheric angular momentum (gray lines, $\Omega a^{2} / 10$ contour interval) averaged over days 150-200 (marked by vertical black lines in panels (a) and (b)).

(A color version of this figure is available in the online journal.) 
(a)
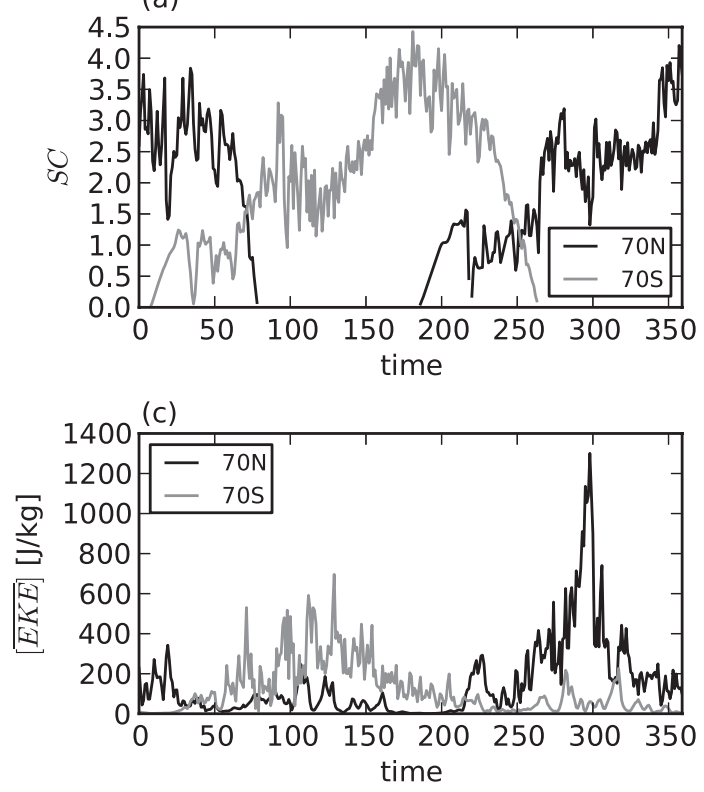

(e)

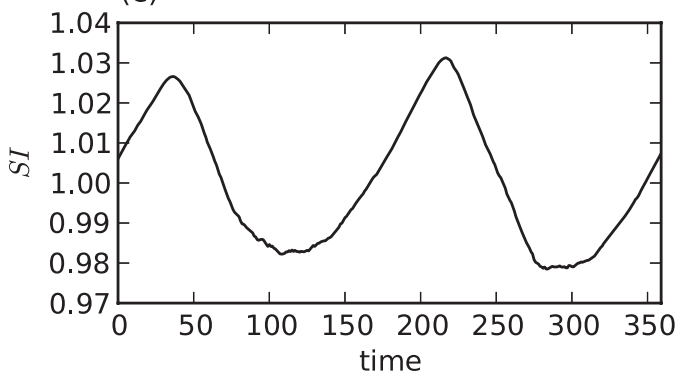

(b) $\quad p_{s}^{\prime}$ at $\max \mathrm{SC}(70 \mathrm{~N})$
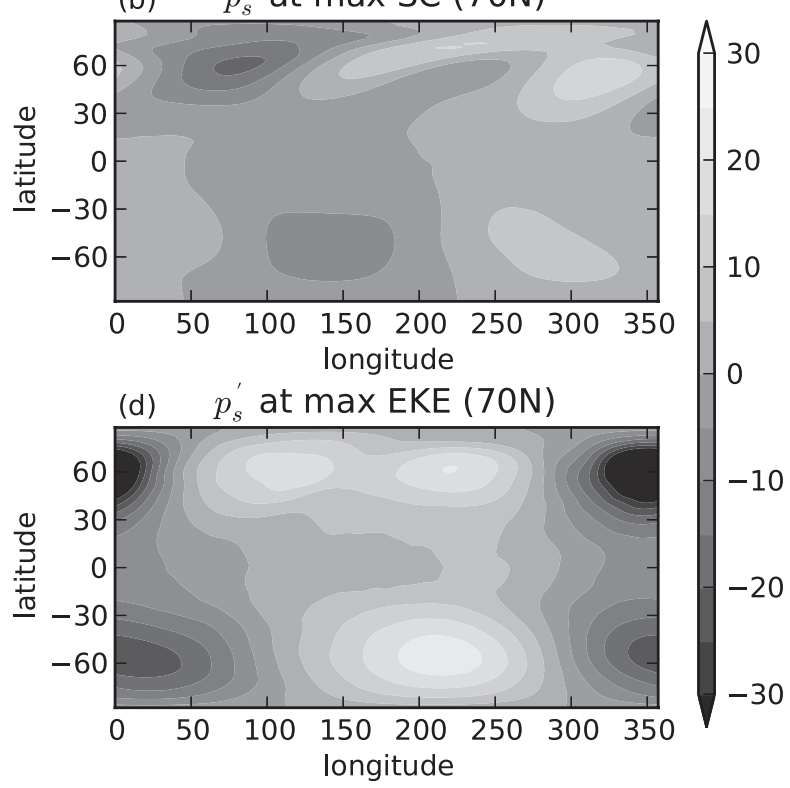

(f)

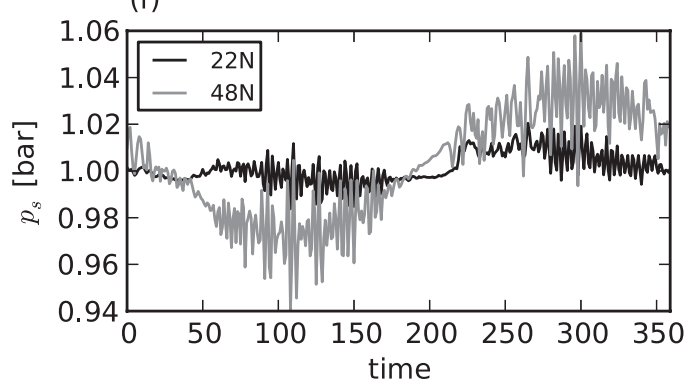

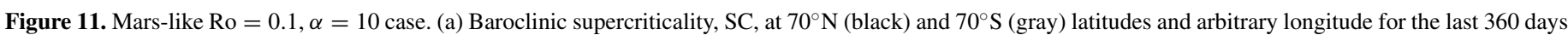

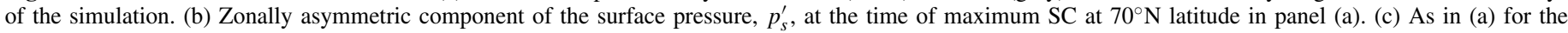

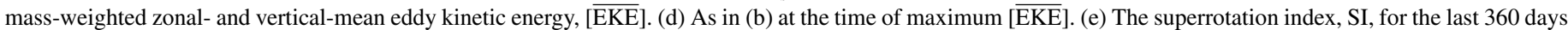
of the simulation. (f) The surface pressure at Viking 1 and Viking 2 latitudes (Hess et al. 1980) and an arbitrary longitude.

our simulation may be due to the fact that we have not included a convection scheme to mimic the release of latent heat in our dry GCM.

A strong, winter Ferrel cell is also quite clear in Figure 10(c), indicating the enhancement of winter-hemisphere baroclinic instability.

\subsection{Mars}

At roughly half Earth's radius and nearly equivalent length of day, Mars is perhaps the most dynamically similar body to Earth with a thermal Rossby number of Ro $\sim 0.1$. The thin Martian atmosphere and solid, dry surface, however, allow large seasonal changes in surface temperature and winds. The surface pressure also varies seasonally on Mars, but this effect is beyond the scope of our idealized approach. For typical values of surface thermal inertia, $I \simeq 100 \mathrm{~J} \mathrm{~m}^{2} \mathrm{~K}^{-1} \mathrm{~s}^{-1 / 2}$ (Putzig et al. 2005), surface temperature, $T_{o} \simeq 220 \mathrm{~K}$, and forcing frequency of Martian year, $\omega \simeq 3 \times 10^{-10} \mathrm{~s}^{-1}$, we find a characteristic value of $\alpha_{\text {Mars }} \simeq 20$ (see the Appendix for derivation). The most Mars-like simulation in our suite is the $\mathrm{Ro}=0.1, \alpha=10$ case.

Mars is known to exhibit rather regular-looking baroclinic eddies with small zonal wavenumber (1-2; Collins et al. 1996), as well as dust storms that are at times global. Relative to Earth, small-wavenumber eddy activity is a natural outcome of Mars' smaller size and thus larger Ro. A less obvious and somewhat counterintuitive feature of the Martian circulation regime is an enhancement in baroclinic SC. Figure 11(a) shows a time series of baroclinic SC at $70^{\circ} \mathrm{N} / \mathrm{S}$ latitudes (black/gray lines) of the last 360 days of the 1080-day simulation. The area of maximum $\mathrm{SC}$ switches from one hemisphere to the other with seasons, peaking in the winter hemisphere at $\sim 4.5$. The counterintuitive aspect is the presence of regular, zonal-wavenumber 1-2 eddies, as evidenced in surface pressure anomalies at the time of peak $70 N-l a t i t u d e$ SC in Figure 11(b). The presence of such regular, wave-like eddies at high values of SC implies that the Rhines scale is larger than the planet, and the inverse energy cascade has been arrested at planetary scales. This dynamical property of Mars' atmosphere may allow it to develop intense mid-latitude "storms" (lacking moisture) at global scales without inducing a turbulent cascade, as observed (Collins et al. 1996).

Figure 11(c) shows the mass-weighted vertical- and zonal-mean eddy kinetic energy, [ $\overline{\mathrm{EKE}}]$, at the same latitudes. There is a positive correlation in time between rising values of SC and the local eddy kinetic energy, which indicates that the winter hemisphere experiences stronger eddy activity. Surface pressure asymmetries are strongest at peak [ $\overline{\mathrm{EKE}}]$, as shown in Figure 11(d). The time series of SI, Figure 11(e), demonstrates an anti-correlation with $[\overline{\mathrm{EKE}}]$ and $\mathrm{SC}$, although low values of SI may also be produced by the strong, negative torque from the cross-equatorial Hadley circulation. 

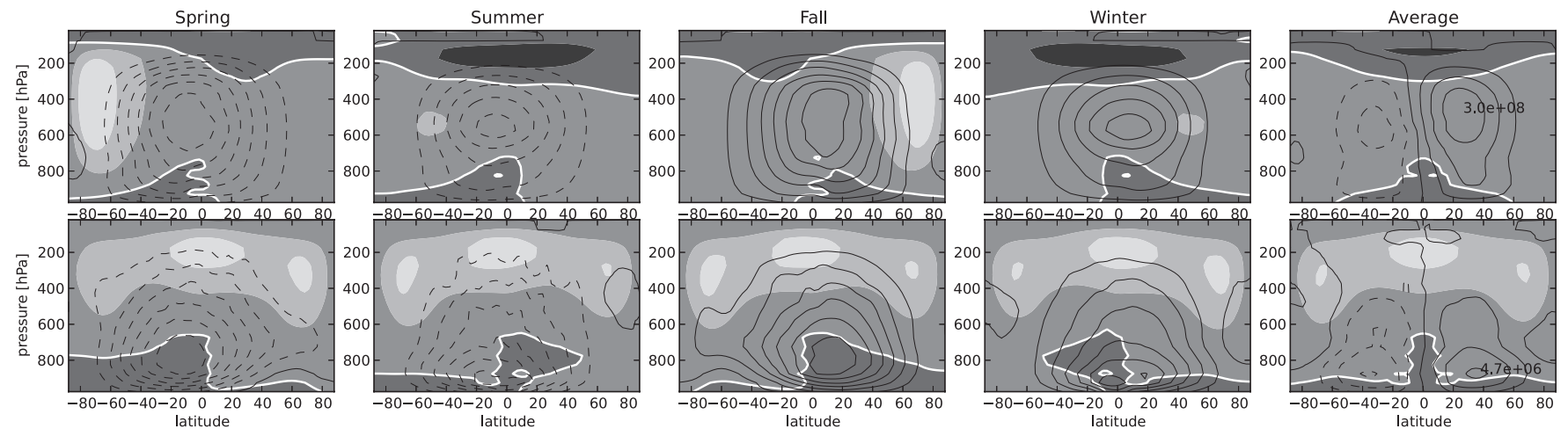

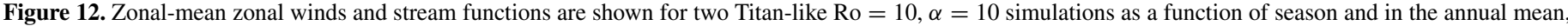

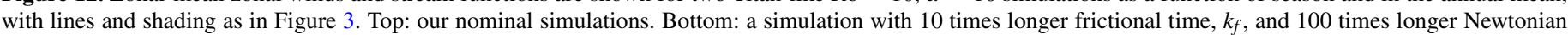
cooling time, $\tau_{r}$

It is noteworthy that despite small values of both SC and $[\overline{\mathrm{EKE}}]$ at $70^{\circ} \mathrm{S}$ latitude (in the summer hemisphere), the disturbances in the surface pressure are seen globally in Figure 11(d) when $[\overline{\mathrm{EKE}}]$ peaks in the winter (northern) hemisphere. Global dust storms are known to occur prior to and during the winter solstice (Hess et al. 1980), and we speculate that this may be similar to the strengthening baroclinicity in the winter hemisphere jet streams in our idealized simulations. In fact, the surface pressure at $70^{\circ} \mathrm{N} / \mathrm{S}$ latitudes (and arbitrary longitude) shown in Figure 11(f) demonstrates that fluctuations by eddies are responsible for $4 \%$ changes, similar to the values observed by the Viking 1 and 2 landers (Hess et al. 1980), albeit at much lower total pressures. Although peaks in eddy activity occur at both solstices in our model, Mars' large hemispheric topographic asymmetry may be responsible for the predominance of global dust storms during the northern winter solstice versus the southern.

\subsection{Titan}

Inspection of the Ro, $\alpha=10$ case in the lower-right panel of Figure 3 reveals that the seasonal cycle has prevented the development of superrotation, thus failing in our primary criterion for "Titan-likeness." Motivated by the extremely long radiative cooling time in Titan's atmosphere, we performed an additional Ro, $\alpha=10$ simulation with 100 times longer atmospheric Newtonian cooling time, $\tau_{r}$, and 10 times weaker boundary layer (Rayleigh) friction, $k_{f}$. Figure 12 displays monthly mean snapshots at each season (left four panels) and the annual average (right panel) of the zonal-mean zonal winds (gray scale, same scale as Figure 3) and meridional stream function (lines) for two Titan-like simulations with Ro, $\alpha=10$. The top row displays the final year of our nominal simulation, while the bottom row shows the final year of a $60 \mathrm{yr}$ simulation with 10 times the frictional time, $k_{f}$, and 100 times the Newtonian cooling time, $\tau_{r}$. In both cases, the Hadley circulation clearly reverses direction with seasons, switching from counterclockwise (dashed lines) in the spring and summer to clockwise (solid lines) in the fall and winter, and is dominated by the cross-equatorial "winter cell." Yet the impact of the Hadley circulation on upper-level winds in the bottom row is muted relative to the top row and appears primarily as an intensification of the winter jet stream. We can understand this by comparing the strength of the meridional stream function (labeled in $\mathrm{kg} \mathrm{s}^{-1}$ in the annual mean), which is 100 times weaker in the bottom row, roughly in inverse proportion to the increased Newtonian cooling time. The sluggish overturning is further apparent in the phase lag of zonal wind accelerations in the bottom versus the top row. A weak Hadley cell limits the negative, seasonal torque on equatorial winds supplied in the form of cross-equatorial advection of momentum at upper levels, allowing equatorial superrotation to persist.

\section{CONCLUSIONS}

Comprehensive planetary GCMs are generally specifically designed to simulate only one particular planetary body, whereas idealized GCMs can, because of their simplicity, in principle be used to study a broad range of atmospheric circulation regimes. Until now, however, idealized GCMs have lacked a generalized seasonal cycle that can be studied over a broad range of planetary regimes. Motivated by the fact that Earth, Mars, and Titan all have pronounced seasonal cycles in insolation, we have developed an idealized, dry atmospheric GCM with a generalized seasonal cycle. The model thermal forcing is that of a Newtonian relaxation toward a specified, time-dependent temperature profile. The time-dependent forcing temperature profile is specified by requiring a slab surface to be in local radiative equilibrium with an idealized form of the seasonal pattern of diurnal-mean insolation. This approach allows the strength of the seasonal cycle of the model simulations to be tuned through a control parameter, $\alpha$, a non-dimensional measure of surface thermal inertia. (Please see Table 1 for a summary of our forcing scheme.)

We then explored the dynamics of the idealized model over a wide range of two control parameters, Ro and $\alpha$, roughly spanning the range that encompasses Earth, Mars, and Titan. We paid particular attention to the parameter regime that allows spontaneous superrotation to emerge. We found that only a relatively small amount of seasonality is necessary to suppress superrotation, apparently for two reasons: (1) the cross-equatorial flow of the Hadley circulation tends to produce equatorial easterlies, and (2) a hemispherically asymmetric zonal wind profile suppresses the global instability that converges momentum on the equator. The extreme sensitivity of our modeled winds to a seasonal cycle demonstrates the importance of the atmospheric thermal inertia, which increases with increasing optical thickness and decreasing temperatures. ${ }^{7}$ In the case of Titan (and Venus), a large atmospheric thermal inertia results in a sluggish Hadley circulation with a long overturn time. The result is a diminished effect of the seasonal cycle on the winds. Indeed, we find

\footnotetext{
7 In the optically thick regime, thermal inertia scales as $\tau_{\text {atm }} \propto \kappa_{\text {ir }} p_{s}^{2} / \bar{T}^{3}$ for an atmosphere with surface pressure $p_{s}$, characteristic infrared opacity $\kappa_{\mathrm{ir}}$, and temperature $\bar{T}$.
} 
that increasing the Newtonian cooling time (a proxy for atmospheric thermal inertia) of our model atmosphere allows strong superrotation to emerge in the presence of seasonal Hadley cell reversals. Through diagnosing baroclinic SC, we also demonstrated that baroclinic instability is a significant impediment to the development of superrotation, presumably through the negative torque in the subtropical "surf zone" from the breaking of equatorward-propagating Rossby waves. The mechanism of the global instability giving rise to equatorial acceleration in our simulations has yet to be identified.

We compared our simulations in parameter regimes relevant to Earth, Mars, and Titan to observed large-scale phenomena on these planets. For the Earth-like regime (Ro $=0.01, \alpha=0.25$; Figure 10), a monsoon-like circulation carries the ITCZ roughly $20^{\circ}$ in latitude into the summer hemisphere. The seasonal cycle in low-level mass flux, however, does not undergo abrupt transitions as in observations. Inspection of the degree of angular momentum conservation in the winter Hadley cell indicates that the nonlinear (inviscid) regime is not achieved in our seasonal model, which disagrees with reanalysis (Bordoni \& Schneider 2008), and the lack of this eddy-mediated regime transition is likely the cause of a relatively smooth seasonal cycle of the ITCZ.

For the Mars-like regime ( $\mathrm{Ro}=0.1, \alpha=10$; Figure 11 ), a very strong seasonal cycle occurs in all diagnostics. Like Mars, this regime exhibits very regular baroclinic waves with zonal wave numbers 1 and 2. Somewhat counterintuitively, the baroclinic SC is quite large in this regime, which should indicate a strong turbulent cascade; however, the Rhines scale is larger than the planetary scale, and so the cascade is arrested. Intense, global storms are a distinct feature of the Mars-like regime, and while their intensities are strongest in the winter hemisphere, the surface pressure perturbations are felt globally. This intensification of winter storms may be analogous to the mechanism responsible for Mars' global dust storms.

For the Titan-like regime ( $\mathrm{Ro}=10, \alpha=10$ ), our model fails to produce strong superrotation. The atmospheric thermal inertia of Titan is much larger than for Earth or Mars, and we find that we can recover strong superrotation in the Titan-like regime by significantly increasing the Newtonian cooling time of the atmosphere, $\tau_{r}$. When this additional parameter is varied, Figure 12 demonstrates that a pronounced seasonal cycle in the Hadley circulation does not prevent the development and persistence of strong superrotation.

J.L.M. acknowledges support from NASA grant No. NNX12AI71G and the IBM Einstein Fellowship from the Institute for Advanced Study. G.K.V. and S.F.P. acknowledge support from the NSF under grant No. AGS-1144302, and G.K.V. also acknowledges the Wolfson Foundation and the Royal Society.

\section{APPENDIX A}

\section{MODEL DESCRIPTION}

\section{A.1. Primitive Equations}

We begin with the primitive equations subject to Rayleigh friction and Newtonian cooling

$$
\begin{aligned}
\frac{\partial u}{\partial t}= & -\frac{u}{a \cos \varphi} \frac{\partial u}{\partial \lambda}-\frac{v}{a} \frac{\partial u}{\partial \varphi}-\omega \frac{\partial u}{\partial p}+\frac{u v}{a} \tan \varphi \\
& +2 \Omega v \sin \varphi-\frac{1}{a \cos \varphi} \frac{\partial \Phi}{\partial \lambda}-r u
\end{aligned}
$$

$$
\begin{gathered}
\frac{\partial v}{\partial t}=-\frac{u}{a \cos \varphi} \frac{\partial v}{\partial \lambda}-\frac{v}{a} \frac{\partial v}{\partial \varphi}-\omega \frac{\partial v}{\partial p}-\frac{u^{2}}{a} \tan \varphi \\
-2 \Omega u \sin \varphi-\frac{1}{a} \frac{\partial \Phi}{\partial \varphi}-r v \\
\frac{\partial \Theta}{\partial t}=-\frac{u}{a \cos \varphi} \frac{\partial \Theta}{\partial \lambda}-\frac{v}{a} \frac{\partial \Theta}{\partial \varphi}-\omega \frac{\partial \Theta}{\partial p}-\frac{\Theta-\Theta_{e}}{\tau_{r}} \\
\frac{\partial \omega}{\partial p}=-\frac{1}{a \cos \varphi} \frac{\partial u}{\partial \lambda}-\frac{1}{a \cos \varphi} \frac{\partial}{\partial \varphi}(v \cos \varphi) \\
\frac{\partial \Phi}{\partial p}=-\frac{R T}{p} .
\end{gathered}
$$

We non-dimensionalize the equations as follows: $t=$ $2 \Omega t^{*} ; \quad p=p^{*} p_{s} ;(u, v)=\left(u^{*}, v^{*}\right) U ; \omega=\omega^{*} U p_{s} / a ; \Phi=$ $\Phi^{*} 2 \Omega U a ;$ and

$$
(\Theta, T)=\left(\Theta^{*}, T^{*}\right) \frac{2 \Omega U a}{R} .
$$

(Note that $U=\left(R T_{0} \Delta_{H} / 2 \Omega a\right)$.) Making the substitutions above into the primitive equations and dropping asterisks without ambiguity,

$$
\begin{aligned}
\frac{\partial u}{\partial t}= & -\operatorname{Ro}\left(\frac{u}{\cos \varphi} \frac{\partial u}{\partial \lambda}+v \frac{\partial u}{\partial \varphi}+\omega \frac{\partial u}{\partial p}+u v \tan \varphi\right) \\
& +v \sin \varphi-\frac{1}{\cos \varphi} \frac{\partial \Phi}{\partial \lambda}-E u \\
\frac{\partial v}{\partial t}= & -\operatorname{Ro}\left(\frac{u}{\cos \varphi} \frac{\partial v}{\partial \lambda}+v \frac{\partial v}{\partial \varphi}+\omega \frac{\partial v}{\partial p}-u^{2} \tan \varphi\right) \\
& -u \sin \varphi-\frac{\partial \Phi}{\partial \varphi}-E u \\
\frac{\partial \Theta}{\partial t}= & -\operatorname{Ro}\left(\frac{u}{\cos \varphi} \frac{\partial \Theta}{\partial \lambda}+v \frac{\partial \Theta}{\partial \varphi}+\omega \frac{\partial \Theta}{\partial p}\right)-\frac{\Theta-\Theta_{e}}{\tau^{*}}
\end{aligned}
$$

$$
\begin{gathered}
\frac{\partial \omega}{\partial p}=-\frac{1}{\cos \varphi} \frac{\partial u}{\partial \lambda}-\frac{1}{\cos \varphi} \frac{\partial}{\partial \varphi}(v \cos \varphi) \\
\frac{\partial \Phi}{\partial p}=-\frac{T}{p}
\end{gathered}
$$

where

$$
\left(\tau^{*}\right)^{-1}=\left(2 \Omega \tau_{o}\right)^{-1}
$$

$$
E=\frac{r}{2 \Omega},
$$

and

$$
\text { Ro }=\frac{U}{2 \Omega a}=\frac{R T_{0} \Delta_{H}}{(2 \Omega a)^{2}}
$$

are non-dimensional numbers governing the flow.

The complete list of non-dimensional numbers is $\tau^{*}, E$, Ro, $\Delta_{h}$, and $\alpha$. 


\section{A.2. Numerical Integration}

We integrate the dry primitive equations using GFDL's Flexible Modeling System (Gordon \& Stern 1982) at T42 resolution with 20 vertical levels spaced evenly in pressure. A fourth-order hyperviscosity is applied to dissipate energy at the grid scale. A small amount of vertical diffusion with a coefficient of $v=0.01 \mathrm{~m}^{2} \mathrm{~s}^{-1}$ acts to smooth grid-scale noise.

\section{APPENDIX B \\ DERIVATION OF $\alpha$ FROM MEASURED THERMAL INERTIA}

We define the thermal inertia of a surface material,

$$
I=\sqrt{\rho c k}
$$

with density $\rho$, specific heat $c$, and thermal conductivity $k$. The skin depth (in $\mathrm{m}$ ) of a sinusoidal thermal wave with frequency $\omega$ is

$$
\delta=\sqrt{\frac{k}{\omega \rho c}} .
$$

From these, we estimate the thermal damping time,

$$
\tau_{f}=\frac{c \rho \delta}{4 \sigma T_{o}^{3}},
$$

and the seasonal control parameter,

$$
\begin{aligned}
\alpha & =\frac{4 \sigma T_{o}^{3} \delta}{k} \\
& =\frac{4 \sigma T_{o}^{3}}{I \sqrt{\omega}} .
\end{aligned}
$$

\section{REFERENCES}

Bordoni, S., \& Schneider, T. 2008, NatGe, 1, 515

Collins, M., Lewis, S., Read, P., \& Hourdin, F. 1996, Icar, 120, 344

dias Pinto, J. R., \& Mitchell, J. L. 2014, Icarus, in press

Forget, F., Hourdin, F., Fournier, R., et al. 1999, JGRE, 104, 24155

Gordon, C. T., \& Stern, W. F. 1982, MWRv, 110, 625

Haberle, R. M., Pollack, J. B., Barnes, J. R., et al. 1993, JGR, 98, 3093

Held, I. M. 2000, in Woods Hole Program in Geophysical Fluid Dynamics (Woods Hole, MA: Woods Hole Oceanographic Institute), 66

Held, I. M., \& Hou, A. Y. 1980, JAtS, 37, 515

Held, I. M., \& Suarez, M. J. 1994, BAMS, 75, 1825

Hess, S., Ryan, J., Tillman, J., Henry, R., \& Leovy, C. 1980, GeoRL, 7, 197

Lebonnois, S., Burgalat, J., Rannou, P., \& Charnay, B. 2012, Icar, 218, 707

Mitchell, J. L. 2008, JGRE, 113, 8015

Mitchell, J. L. 2012, ApJL, 756, L26

Mitchell, J. L., Pierrehumbert, R. T., Frierson, D. M., \& Caballero, R. 2006, PNAS, 103, 18421

Mitchell, J. L., Pierrehumbert, R. T., Frierson, D. M., \& Caballero, R. 2009, Icar, 203, 250

Mitchell, J. L., \& Vallis, G. K. 2010, JGR, 115, E12008

Newman, C. E., Lee, C., Lian, Y., Richardson, M. I., \& Toigo, A. D. 2011, Icar, 213, 636

Pierrehumbert, R. T., \& Swanson, K. L. 1995, AnRFM, 27, 419

Plumb, R. A., \& Hou, A. Y. 1992, JAtS, 49, 1790

Potter, S. F., Vallis, G. K., \& Mitchell, J. L. 2013, JAtS, 71, 596

Putzig, N. E., Mellon, M. T., Kretke, K. A., \& Arvidson, R. E. 2005, Icar, 173,325

Randel, W. J., \& Held, I. M. 1991, JAtS, 48, 688

Rannou, P., Montmessin, F., Hourdin, F., \& Lebonnois, S. 2006, Sci, 311, 201

Read, P. L. 2011, P\&SS, 59, 900

Saravanan, R. 1993, JAtS, 50, 1211

Schneider, T. 2004, JAtS, 61, 1317

Schneider, T., \& Bordoni, S. 2008, JAtS, 65, 915

Schneider, T., Graves, S., Schaller, E., \& Brown, M. 2012, Natur, 481, 58

Sobel, A. H., \& Bretherton, C. S. 2000, JCli, 13, 4378

Suarez, M. J., \& Duffy, D. G. 1992, JAtS, 49, 1541

Tokano, T., Neubauer, F. M., Laube, M., \& McKay, C. P. 2001, Icar, 153,130

Vallis, G. K. 2006, Atmospheric and Oceanic Fluid Dynamics (Cambridge: Cambridge Univ. Press), 745

Wilson, R. J. 1997, GeoRL, 24, 123 